BENTHAM OPEN
CrossMark
Content list available at: www.benthamopen.com/TOUNJ/
DOI: $10.2174 / 1874303 \times 01609010007$

\title{
Management of Acute and Chronic Gout - The Nephrology Perspective
}

\author{
Syed M. Ahmed and James L. Bailey ${ }^{*}$ \\ Nephrology Fellowship Program, Division of Nephrology, WMB Research Building, 1639 Pierce Drive, Emory \\ University School of Medicine, Atlanta, GA 30322, USA
}

\begin{abstract}
Background: Gout and its treatment pose a greater burden on patients with chronic kidney disease (CKD). We review the incidence of hyperuricemia in patients with $\mathrm{CKD}$, mechanism of urate handling by the kidney and management of acute and chronic gout in patients with CKD and on renal replacement therapy.

Renal Handling of Urate: Reabsorption of urate is enhanced by the presence of monocarboxylate anions. URAT 1 is the predominant urate-anion exchanger followed by the GLUT 9 transporter. Various drugs inhibit these transporters. NPT1 and NPT4 are transporters which secrete urate and NPT 4's action is inhibited by diuretics. Alcohol, volume depletion, salt restriction and high PTH levels cause hyperuricemia.

Management of Gout in CKD: For acute gout attack, NSAIDs are relatively contraindicated. Lower doses of colchicine are recommended in CKD and even lower doses for ESRD patients. Glucocorticoids are the preferred agents in renal patients. In chronic gout, the goal is to maintain uric acid levels $<6 \mathrm{mg} / \mathrm{dl}$. Prophylactic colchicine is recommended for 3 months in patients without tophi and 6 months for patients with tophi. Allopurinol, at doses ranging from 100 to $300 \mathrm{mg} / \mathrm{day}$, lowers serum uric acid levels, but it has not been shown to slow CKD progression. Safety data for Febuxostat is lacking in advance CKD, while Probenecid is in effective in patient with GFR $<30 \mathrm{ml} / \mathrm{min}$. Dose reduction for Pegloticase is not necessary.
\end{abstract}

Keywords: Gout treatment in CKD, renal handling of urate.

\section{INTRODUCTION}

Gout is a painful inflammatory arthritis that affects the general population. However its morbidity and consequences of its treatment poses a greater healthcare burden on patients with chronic kidney disease. Numerous studies have demonstrated that hyperuricemia is associated with a reduced estimated GFR and one study has suggested that adults with eGFR $<60 \mathrm{ml} / \mathrm{kg} / 1.73 \mathrm{~m} 2$ are at 2 -fold increased risk of developing hyperuricemia. Animal and human studies suggest that urate may play a significant role in cardiovascular disease [1], hypertension [2], and progression of renal disease [3]. The latter is controversial. Murray and Goldberg [4] suggested that $11 \%$ of the cases of chronic interstitial nephritis were primarily due to disorders of uric acid metabolism. However, Yu and Berger [5] could not demonstrate that there was such an association and felt that chronic interstitial nephritis could be attributed to hypertension, stones, aging and vascular disease. Whether chronic hyperuricemia alone can lead to interstitial disease is controversial. This is important as so many patients with chronic kidney disease have serum uric acid levels above $10 \mathrm{mg} / \mathrm{dl}$. This is attributed to either declining glomerular filtration rate or to diuretics. The 2007-2008 data from NHANES revealed that 8.3 million Americans are affected by gout, of which $71 \%$ had CKD stage greater than stage 2 (GFR $<60$ ). The prevalence of CKD stage 3 or greater in gout was $19.9 \%$. Inclusion of proteinuria in the definition of CKD has led to investigation of prevalence of gout in patients with proteinuria. Juraschek et al. [6] found a graded positive relationship between gout and albuminuria and Krishnan et al. [7] stated that proteinuria is independently associated with increased

\footnotetext{
* Address correspondence to this author at the Nephrology Fellowship Program, Division of Nephrology, WMB Research Building, 1639 Pierce Drive, Emory University School of Medicine, Atlanta, GA 30322, USA; Tel: 404-727-2525; E-mail: jlbaile@emory.edu
} 
risk of gout. Studies have shown that 5\% of dialysis patients develop gout in the first year of dialysis and $15.4 \%$ will develop gout within the first 5 years of dialysis. Although reduced kidney function can precede the development of gout [8], gout may also adversely impact renal function. This has prompted several studies that have evaluated the adequacy of treatment of gout [9]. Many have found that the treatment of gout within the CKD population to be sub-optimal. For example, the German CKD study [9] reported about 30\% of CKD patients with gout received no uric acid lowering medications. Here we will review the mechanism of renal urate handling and management of gout in the CKD population.

\section{URIC ACID AND THE KIDNEY}

The kidney is responsible for more than $70 \%$ of urate excretion. It has an enormous capacity for urate secretion and the hyperuricemia in gout most commonly results from an undersecretion rather than overproduction of uric acid. Multiple studies have evaluated the transporters involved in the renal handling of urate, and genome-wide association studies have now provided a better understanding of the molecular mechanism of urate handling. Human studies have shown that urate in plasma is minimally bound to proteins and almost $100 \%$ freely filterable at the glomerulus into the renal tubule. In steady state only $5 \%$ to $10 \%$ of filtered urate is excreted in the urine. Interestingly, the kidney maintains this excretion rate in the face of an increased urate concentration, which underscores the remarkable capacity of the kidney to increase its urate reabsorption. Moreover, $99 \%$ of the filtered urate can be reabsorbed even if the filtered load is increased fourfold [10].

Renal Urate Reabsorption: Sodium dependent absorption of lactate and other monocarboxylate anions increases the intracellular concentration of anions that exchange with luminal urate. High levels of circulating levels of lactate, ketones, nicotinate and pyrazinoate result in increased uptake at the apical membrane and a secondary increase in apical urate-anion exchange, which results in hyperuricemia. URAT1 is the predominate urate-anion exchanger in the luminal membrane of the proximal tubule. It is localized to the brush border membranes of renal proximal tubule cells. URAT1 is inhibited by uricosuric agents such as probenecid and benzbromarone as well as by losartan. GLUT 9 is a voltagedependent urate transporter located on the basolateral membrane of proximal tubule. Both URAT1 and GLUT9 are inhibited by losartan in vitro. It has been observed clinically that this effect is not a drug class effect but is specific to losartan, as the oocyte studies have shown that URAT1 and GLUT9 are inhibited by losartan and not by valsartan [11].

Renal Urate Secretion: ABCG2, adenosine triphosphate binding cassette transporter 2, is a multidrug resistant transporter that is localized in the apical membrane of the proximal tubular cells and mediates urate secretion across the apical membrane [12]. NPT1 (Sodium phosphate transporter 1) is located in the proximal tubule apical membrane and has been shown to transport both phosphate and urate. The NPT4 (Sodium phosphate transporter 4) has also been shown to be localized in the apical membrane of renal proximal tubule cells, and to transport urate. Diuretics interact with NPT4 and alter renal urate handling. Both thiazide and loop diuretics can directly inhibit NPT4-mediated urate secretion.

Factors that affect serum urate level: Exposure to high altitude increases serum urate levels. Alcohol intake increases serum lactate and beta-hydroxy butyrate levels causing indirect activation of renal urate reabsorption, increasing serum urate levels. Volume depleted state causes hyperuricemia. Both short term and long term salt restriction in humans causes significant hyperuricemia, which is dramatically reversed by salt loading. Excessive parathyroid hormone levels reduce urate excretion in primary hyperparathyroidism, and urate excretion is also reduced during the pharmacological treatment of osteoporosis [13].

\section{MANAGEMENT OF ACUTE GOUT IN PATIENTS WITH CHRONIC KIDNEY DISEASE}

Gouty arthritis patients have multiple co-morbidities. For example, review of the Veterans Affairs medical database showed that $47 \%$ had chronic kidney disease [13]. The National Health and Nutrition Examination Survey [13] revealed that $9 \%$ of patients with gouty arthritis had renal impairment. As noted previously, it is not clear if hyperuricemia plays a role in the pathogenesis of CKD or if it is simply a marker of the disease. Nevertheless the management of gouty arthritis requires a dual approach: management of inflammation and management of hyperuricemia. Typically treatment of inflammation is reserved for acute gouty attacks, but anti-inflammatory therapy may also be needed to control chronic low grade inflammation during inter-attack periods. Urate lowering therapy is necessary to maintain uric acid levels below its solubility level $(<6 \mathrm{mg} / \mathrm{dl})$ in order to prevent acute gout attacks. Treatment choices in the management of acute gout are as follows. 


\section{NSAIDS}

EULAR (European League Against Rheumatism) guide-lines [16] and recommendations for NSAIDs are as follows for the different stages of chronic kidney disease: NSAIDs are relatively contraindicated in CKD stage I-II. If they are used, the suggested dose is indomethacin $50 \mathrm{mg}$ three times a day or an equivalent NSAID until pain becomes tolerable. It is not to be used for more than a week, and the dose should be rapidly reduced to zero. Caution is advised in CKD stage III. Creatinine levels should be monitored carefully. NSAIDS are generally not recommended in stage IV-V because of the adverse effect they can have on renal function.

\section{Colchicine}

In patients with CKD, low dose colchicine given within 36 hours of onset of symptoms is equivalent to high dose colchicine. It is usually given orally in doses of $1.2 \mathrm{mg}$ followed by an additional dose of $0.6 \mathrm{mg}$ in $1 \mathrm{hr}$. This treatment should be repeated no more than once every 2 weeks. For patients requiring more than the recommended doses of colchicine, alternate therapy should be considered. Patients with a GFR less than $80 \mathrm{ml} / \mathrm{min}$ should be monitored closely because it can adversely affect renal function. For patients with ESRD, a single dose of colchicine of $0.6 \mathrm{mg}$ is recommended and treatment should not be repeated more than once every 2 weeks.

\section{Glucocorticoids}

There are limited studies on the use of corticosteroids in the treatment of acute gout; however, corticosteroids are the preferred treatment for acute gouty attacks in patients with CKD. EULAR guidelines suggest that the route of administration be an intra-articular injection of long acting steroids. Theoretically, this is done to reduce the systemic effect of steroids, but complications such as hyperglycemia can and still do occur.

\section{MANAGEMENT OF CHRONIC GOUT IN PATIENT WITH CKD}

Lowering serum uric acid levels to less than $6 \mathrm{mg} / \mathrm{dl}$ in CKD patients with gout has a tendency to reduce acute gouty flares. The presence of CKD does not alter the need to reach this goal; however, levels less than $5 \mathrm{mg} / \mathrm{dl}$ may be more appropriate in advance disease. Treatment options in the treatment of chronic gouty arthritis for lowering serum uric acid levels are as follows:

\section{Colchicine}

A prophylactic dose of colchicine for gout in patients with severe renal impairment should be $0.3 \mathrm{mg} / \mathrm{d}$. Any increase in dose warrants caution and requires regular monitoring of renal function. A starting dose of Colchicine 0.6 mg PO BID co-administered with xanthine oxidase inhibitors has been shown to reduce acute gouty flares. Current data supports the administration of colchicine after achieving a serum uric acid level less than $6 \mathrm{mg} / \mathrm{dl}$ after 3 months in patients without tophi and 6 months in patients with tophi. The recommended dose in patients with ESRD is $0.3 \mathrm{mg}$ twice a week.

\section{Allopurinol}

EULAR guidelines suggest that allopurinol be used to lower uric acid levels for the long term. Although allopurinol is excreted in the urine as its metabolite, oxypurinol, which can accumulate to toxic levels in patients with renal impairment and has been implicated in allopurinol associated hypersensitivity syndrome (AHS). Studies have shown that AHS is variably dose dependent and does not always correlate with oxypurinol levels. The current recommendations for dosing of allopurinol are to minimize the risk of AHS and to avoid gouty flares. Patients with CKD stage I and II should be monitored closely for renal disease progression. In CKD stages III-V, the starting dose is $50 \mathrm{mg} /$ day; it can gradually be increased up to $300 \mathrm{mg} /$ day in order to reach a goal of a serum uric acid level of $6 \mathrm{mg} / \mathrm{dl}$ or less. It has been proposed that the urate-lowering effects of allopurinol may slow the progression of CKD; however, adequately powered randomized controlled trials are necessary to confirm this.

\section{Febuxostat}

The recommended dose is $40 \mathrm{mg}$ or $80 \mathrm{mg} / \mathrm{d}$. As there is a lack of published safety data for febuxostat in the setting of CKD stage IV or higher, it is not known whether dose adjustments are necessary in advance CKD. No dose adjustments are required for GFR $>30 \mathrm{ml} / \mathrm{min}$. At least 1 study has shown that the urate lowering effects of febuxostat correlated with stabilization or slowing the progression of CKD $[14,15]$. 


\section{Probencid}

The recommended starting dose is $250 \mathrm{mg}$ twice daily. After one week, the dose can be increased to $500 \mathrm{mg}$ twice a day. The maximum dose is $2000 \mathrm{mg}$ /day. It is ineffective in patients with a GFR $<30 \mathrm{ml} / \mathrm{min}$ because it inhibits the reuptake of uric acid in the proximal tubule. It is also contraindicated in patients with renal stones.

\section{Pegloticase}

A pegylated uricase has been approved for the treatment of chronic gouty arthritis in patients refractory to conventional therapy. The recommended dose is $8 \mathrm{mg}$ as an intravenous infusion every 2 weeks. No dose reduction has been recommended in patients with CKD, but pegloticase has not been formally studied in patients with CKD.

\section{MANAGEMENT OF GOUT IN PATIENTS WITH RENAL TRANSPLANT}

\section{Acute Gouty Attacks}

NSAIDs are generally avoided after renal transplantation. Although Sulindac or nonacetylated salicylates are theoretically renal sparing, they still should be used with caution in this population. Renal function should be monitored weekly. At low doses, salicylates can cause elevation in uric acid levels. At high doses, they act as uricosuric agents.

Corticosteroids: These are the preferred agents for treatment of acute gout in the setting of renal transplantation. As most transplant patients are on maintenance dose of prednisone, a higher dose is required. Usually, the dose of prednisone is increased to 0.5 to $1 \mathrm{mg} / \mathrm{kg}$ per day for 3 to 7 days and then tapered to the maintenance dose. In patients who are intolerant of higher doses of steroids, ACTH intra-articular injection 40 - 80 IU is a reasonable alternative[17].

Colchicine: Commonly reported side effects of colchicine include abdominal cramps, nausea and diarrhea which can be seen in patients taking mycophenolate. A starting dose of $0.6 \mathrm{mg}$ every six hours and then titrated to avoid diarrhea is reasonable. A more serious adverse side effect associated with colchicine is myoneuropathy. The Cleveland Clinic group has recommended that colchicine be given in divided doses ( $0.6 \mathrm{mg}$ BID) on days 1 and 2 , followed by 0.6 $\mathrm{mg}$ once daily for days 3-10. The drug can then be stopped for patients with biopsy proven myoneuropathy. Colchicine should be held if patients develop diarrhea.

\section{CONFLICT OF INTEREST}

The authors confirm that this article content has no conflict of interest.

\section{ACKNOWLEDGEMENTS}

Declared none.

\section{REFERENCES}

[1] Feig DI, Kang DH, Johnson RJ. Uric acid and cardiovascular risk. N Engl J Med 2008; 359(17): $1811-21$. [http://dx.doi.org/10.1056/NEJMra0800885] [PMID: 18946066]

[2] Mazzali M, Hughes J, Kim YG, et al. Elevated uric acid increases blood pressure in the rat by a novel crystal-independent mechanism. Hypertension 2001; 38(5): 1101-6. [http://dx.doi.org/10.1161/hy1101.092839] [PMID: 11711505]

[3] Mazzali M, Kanellis J, Han L, et al. Hyperuricemia induces a primary renal arteriolopathy in rats by a blood pressure-independent mechanism. Am J Physiol Renal Physiol 2002; 282(6): F991-7. [http://dx.doi.org/10.1152/ajprenal.00283.2001] [PMID: 11997315]

[4] Murray T, Goldberg M. Chronic interstitial nephritis: etiologic factors. Ann Intern Med 1975; 82(4): $453-9$. [http://dx.doi.org/10.7326/0003-4819-82-4-453] [PMID: 1119762]

[5] Yü TF, Berger L. Impaired renal function gout: its association with hypertensive vascular disease and intrinsic renal disease. Am J Med 1982; 72(1): 95-100.

[PMID: 7058827]

[6] Juraschek SP, Kovell LC, Miller ER III, Gelber AC. Association of kidney disease with prevalent gout in the United States in 1988-1994 and 2007-2010. Semin Arthritis Rheum 2013; 42(6): 551-61. [http://dx.doi.org/10.1016/j.semarthrit.2012.09.009] [PMID: 23312548]

[7] Krishnan E. Reduced glomerular function and prevalence of gout: NHANES 2009-10. PLoS One 2012; 7(11): e50046. [http://dx.doi.org/10.1371/journal.pone.0050046] [PMID: 23209642] 
[8] Krishnan E. Chronic kidney disease and the risk of incident gout among middle-aged men: a seven-year prospective observational study. Arthritis Rheum 2013; 65(12): 3271-8. [http://dx.doi.org/10.1002/art.38171] [PMID: 23982888]

[9] Titze S, Schmid M, Köttgen A, et al. GCKD study investigators. Disease burden and risk profile in referred patients with moderate chronic kidney disease: composition of the German Chronic Kidney Disease (GCKD) cohort. Nephrol Dial Transplant 2015; 30(3): 441-51. [http://dx.doi.org/10.1093/ndt/gfu294] [PMID: 25271006]

[10] Levinson DJ, Sorensen LB. Renal handling of uric acid in normal and gouty subject: evidence for a 4-component system. Ann Rheum Dis 1980; 39(2): 173-9.

[http://dx.doi.org/10.1136/ard.39.2.173] [PMID: 7387222]

[11] Lipkowitz MS. Regulation of uric acid excretion by the kidney. Curr Rheumatol Rep 2012; 14(2): $179-88$. [http://dx.doi.org/10.1007/s11926-012-0240-z] [PMID: 22359229]

[12] Woodward OM, Köttgen A, Coresh J, Boerwinkle E, Guggino WB, Köttgen M. Identification of a urate transporter, ABCG2, with a common functional polymorphism causing gout. Proc Natl Acad Sci USA 2009; 106(25): 10338-42. [http://dx.doi.org/10.1073/pnas.0901249106] [PMID: 19506252]

[13] Mount DB. The kidney in hyperuricemia and gout. Curr Opin Nephrol Hypertens 2013; 22(2): $216-23$. [http://dx.doi.org/10.1097/MNH.0b013e32835ddad2] [PMID: 23318701]

[14] Whelton A, Macdonald PA, Zhao L, Hunt B, Gunawardhana L. Renal function in gout: long-term treatment effects of febuxostat. J Clin Rheumatol 2011; 17(1): 7-13. [http://dx.doi.org/10.1097/RHU.0b013e318204aab4] [PMID: 21169856]

[15] Curiel RV, Guzman NJ. Challenges associated with the management of gouty arthritis in patients with chronic kidney disease: a systematic review. Semin Arthritis Rheum 2012; 42(2): 166-78. [http://dx.doi.org/10.1016/j.semarthrit.2012.03.013] [PMID: 22560299]

[16] Khanna D, Fitzgerald JD, Khanna PP, et al. American College of Rheumatology. 2012 American College of Rheumatology guidelines for management of gout. Part 1: systematic nonpharmacologic and pharmacologic therapeutic approaches to hyperuricemia. Arthritis Care Res (Hoboken) 2012; 64(10): 1431-46. [http://dx.doi.org/10.1002/acr.21772] [PMID: 23024028]

[17] Khanna D, Khanna PP, Fitzgerald JD, et al. American College of Rheumatology. 2012 American College of Rheumatology guidelines for management of gout. Part 2: therapy and antiinflammatory prophylaxis of acute gouty arthritis. Arthritis Care Res (Hoboken) 2012; 64(10): $1447-61$. [http://dx.doi.org/10.1002/acr.21773] [PMID: 23024029]

Received: April 18, 2015 Revised: April 30, 2015 Accepted: April 30, 2015

(C) Ahmed and Bailey; Licensee Bentham Open.

This is an open access article licensed under the terms of the Creative Commons Attribution-Non-Commercial 4.0 International Public License (CC BY-NC 4.0) (https://creativecommons.org/licenses/by-nc/4.0/legalcode), which permits unrestricted, non-commercial use, distribution and reproduction in any medium, provided the work is properly cited. 